\title{
Trademarks' License Agreement Based on a Smart Contract
}

\author{
Odintsov S.V. ${ }^{*}$ Mansour M.
}

\author{
RUDN University, Moscow, Russia \\ *Corresponding author. Email: odintsov_sv@pfur.ru
}

\begin{abstract}
The subject of this paper is the review of the feasibility and advisability of using smart contracts based on blockchain technologies for the legal regulation of relations to provide the exclusive rights to means of individualization (trademarks). Blockchain technology is one of the very promising fields in the area of digitalization of the economy, which continues to be constantly and actively developed. The use of selfexecutable transactions by subjects of civil (commercial) circulation is becoming increasingly relevant. However, it should be noted that law enforcement practice has yet to be formed in this area.

The existing civil (commercial) law has recently begun to develop provisions defining the specifics of the circulation of so-called digital assets, which may also include exclusive rights and the results of intellectual activity, including trademark rights.

So far, the existing legal regulation regarding the rights to digital assets (tokens) and the introduction of smart contracts into the circulation can hardly be called sufficient. The paper discusses the rationale for the feasibility and advisability of using smart contracts for registration of license agreements regarding the use of trademark rights. As a result of the study and practical proposal, the authors focus attention on the need for an internationally unified approach to using blockchain technologies for fixing the transfer (assignment) of exclusive rights to the results of intellectual activity.
\end{abstract}

Keywords: blockchain technologies, token, smart contract, digital assets, trademarks, license agreement

\section{INTRODUCTION}

It is unpromising, and as well generally impossible, to argue with the fact that scientific and technological progress dramatically affects the economy in general, the area of consumption and specifics of the relations between the subjects of circulation. As a consequence of the ongoing digitalization processes at the present stage, this is not only a doctrinal understanding but also the regulatory formulation of new types of property rights and obligatory legal relations for all law and order.

The modern material content of the economy, property circulation, is already being transformed dramatically under the influence and in the implementation of digital technologies. Within the framework of the digital "revolution," the border between users of these technologies and their creators and copyright holders has practically vanished since digital technologies are not as much used tools but primarily processes that subjects adapt to their needs and thus transform, modify, and develop [1].

The introduction of new technologies will improve the operation of the infrastructure, increase its safety, reliability and efficiency [2]. Blockchain technologies are already in the phase of active adoptions by the corporate mainstream, the grade of adoption to increase and cover virtually all areas of our lives. Hence it is important to

analyze trends and define its prospective developments [3].

At the same time, and under the conditions of the digital economy, there remains a need for fixing exclusive rights to means of individualization of subjects and objects of circulation and, first of all, trademarks (service marks, appellations of origin), in the integrated management of the intellectual property. This need will only increase since trademarks are means of individualization in commercial circulation that allow a potential consumer to identify and distinguish an attractive (acceptable, required) product or service.

\section{METHODOLOGY}

A comprehensive review of the possibility and advisability of using smart contracts based on blockchain technologies for the legal regulation of relations to provide the exclusive rights to means of individualization (trademarks), including an analysis of the law enforcement 
practice of tokenizing the global economy, including legal regulation of registration and use of exclusive rights to means of individualization of subjects and objects of circulation and, first of all, trademarks, all these and related legal aspects in different jurisdictions, is possible only when using a comparative legal method of study.

At the same time, it is important to pay attention to the positions of experts in various areas, to study trends and problems in the application and development of blockchain technologies, which potentially allow creating and providing a reliable digital architecture for the legal protection of the intellectual property rights.

\section{RESULTS}

Based on the fact that the emergence of new digital technologies creates confusion in terms of the application of traditional laws on property rights in the digital sphere. Therefore, a more simplified model of ownership, which protects the integrity of the goods and services used by consumers, becomes advisable. It is the introduction of blockchain technologies that will allow the subjects of property circulation to own and fully use their rights to both conventional real estate items and intangible digital assets, which may mediate the intellectual property as well [4]. One of the main problems for copyright holders regarding digital assets, typically, is the existing high risk of their illegal copying (use). The use of smart contracts, blockchain algorithms, and associated technology of "distributed accounting" offers copyright holders obvious opportunities for legal protection and protection of property rights, including evidence of authentication and the fact of the first use either at the stage of registration of rights or in court proceedings.

The digital technological innovations developed into legal novelties, such as the blockchain, which is the essence of transparency [3]. The practice of using network smart contracts is accepted and taken into account as the standard today in many law and order. However, it is also worth noting the completely reasonable caution of introducing legal regulation regarding the intellectual property. Particularly, it should be noted that at the moment, there is not even a uniform regulatory approach to the basic concepts of the blockchain technology mediating the presence of property rights of the subjects of circulation.

Of course, it is impossible to deny the fact that the blockchainization is steadily affecting more and more areas of civil (commercial) circulation, while tokens (digital assets) possess the characteristic of full-fledged objects of law, or it contributes to mediating the corresponding objects of law digitally.

The absence of a unified concept of the essence of the tokenization, respectively, entails current problems, in which each law and order has its own peculiarities of regulatory formulation and doctrinal interpretation of the concept of the essence and legal nature of tokens. In any case, it is worth starting from the fact that tokens undoubtedly represent a special (digital) way of fixing the right to civil (commercial) law objects, including the intellectual property, and asset tokenization processes will only intensify [5]. Therefore, in order to optimize the circulation of assets, including within the framework of the globalization of financial markets, it is extremely important for subjects to have a uniform regulatory concept for the use of blockchain technologies, unified by the relevant international legal act.

It should be based on the understanding that smart contracts cannot be deprived of legal force, validity, or enforcement only because the contract is processed, executed, or otherwise applied through an intellectual agreement, a computer code.

\section{DISCUSSION}

It is hard to overestimate the importance of means of individualization, which not only serves as a way of distinction but also with the help of which, and sometimes exclusively with the help of which, the choice of contractors, goods (services) takes place. This applies equally to all participants in the civil circulation, either businessmen or ordinary consumers. Means of individualization are a kind of link between the manufacturer and the consumer. In the absence of means of individualization, it is impossible to draw the attention of potential consumers to certain goods (services), as well as it is also impossible to choose the required goods (services) of appropriate quality for consumers. Therefore, the presence of certain gaps in the legislation leads to a violation of the exclusive rights and legitimate interests of copyright holders.

In the process of mass distribution of information technologies, the civil (commercial) circulation begins to be fully formed in the virtual space of the digital environment. Currently, information (digital) technologies, through which more and more various transactions are concluded, are not adequately subject to legal regulation either by international law or by national legislation and judicial practice. In the process of developing in recent years, blockchain technologies continue to experience a real burst of commercialization with an ever-increasing amount of funds raised. Self-executable transactions (smart contracts) are successfully applied in various jurisdictions; the process of regulatory legalization of relations for used means of individualization (trademarks) is expanding.

Traditionally, the following types of licensing agreements on the provision of the right to use trademarks are found in the professional literature:

- exceptional - a trademark is used only by a person who has received this exclusive (exclusive) right during a certain period;

- simple - more than one licensee has the right to use since the owner of the mark, the licensor, is entitled to conclude licensing agreements with other users; 
- sublicenses - by virtue of which the licensee may itself grant a license to third parties;

- full - when the right to use a trademark (service mark) applies to all goods (services), in respect of which the mark is registered; and

- partial - when the right to use a trademark (service mark) applies only to a part of the goods (services), for which the mark is registered.

Moreover, all of the above types of license agreements may optimally be drawn up based on smart contracts using blockchain technologies. Of course, it is impossible to proceed from some kind of global universality of forms of self-executable transactions (smart contracts) in view of the fact that they may vary significantly depending on the type of the corresponding blockchain platform, basis on and within which they are applied.

In principle, the use of smart contracts based on the technology of decentralized distributed registries (databases) entails a number of positive aspects, such as minimizing transaction costs and a high level of security and confidentiality, including through the decentralization and the use of cryptographic protection.

It is also worth mentioning that blockchain technologies may help initially fix the rights of the copyright holder to the corresponding trademark. In this case, the process of tokenization of the intellectual property takes place. From an economic point of view, the significance and value of tokens lies in the fact that tokens may be a conditional (accountable, in the corresponding system) unit of a certain value and thereby directly serve as a digital asset, and at the same time, tokens may also be and a shell with the goal of digitally mediating a specific asset (item, property rights, intangible values, results of work or services, and results of intellectual activity, including means of individualization). In this case, such tokens should be considered as the so-called "secured tokens" fixing the rights to other objects or as "certificate tokens" providing an opportunity to mediate the rights of their owner to items (movable and immovable), results of work/ services, securities, shares in the authorized capital, as well as other tangible and intangible objects, since such a token acts as a virtual image, a symbol of property, in respect of which legally significant actions are performed.

A smart contract is executable code that runs on the blockchain to facilitate, execute and enforce the terms of an agreement. The main aim of a smart contract is to automatically execute the terms of an agreement once the specified conditions are met [6]. Smart contracts, first of all, differ from "conventional" ones in that the parties fulfill their obligations thereunder "automatically," without the influence of third parties, including state authorities, as well as legislative regulation and administration. But at the same time, the consequences of improper fulfillment or non-fulfillment of obligations apparently also require coercion or application of appropriate sanctions on the part of the court if the smart contract itself does not contain the regulation laid down by the parties to ensure the proper fulfillment of obligations and liability of the parties.
Through the use of appropriate computer information platforms (resources), a form of concluding contracts by means of certain specific actions performed by subjects is becoming more common: clickwrap agreement (agreement concluded by clicking the mouse) and browsewrap agreement (agreement concluded by using the website) [7]. UNCITRAL model law on electronic commerce 1966 equates electronic contracts and the procedure for their conclusion (particularly, through the clickwrap agreement) with written contracts in terms of legal force [8].

The certainty regarding the use of self-executable transactions (smart contracts), which are not a separate independent transaction, is only a condition for the automatic execution of any civil law contract.

On the one hand, expression of will using electronic or other similar technical means is equivalent to a simple written form of a transaction (provided that it is possible to reproduce the contents of the transaction unchanged on a physical medium (for example, print it on paper) and reliably identify counterparties (for example, use biometric identification. The expression of will is carried out by the parties through electronic signatures using cryptographic keys to ensure confidentiality [9].

Of primary importance for self-executable transactions based on blockchain technologies is the initial certainty and agreement by the parties of the authenticity of their will regarding the essential terms of the contract because in the future, in the case of a dispute, it will be quite difficult to establish and interpret for the purposes of judicial (arbitration, mediation) proceedings the intentions of the parties recorded in decentralized distributed registries.

For several years now, blockchain-based smart contracts for registers (cadastres) of rights to real estate and transactions with them have been successfully used in a number of jurisdictions. It is the blockchain principles and technologies of decentralized distributed registries that allow achieving ideal confidentiality and cryptographic authentication and verification of the facts of the fulfillment of the terms and conditions of smart contracts by the parties. Among other things, self-executable transactions significantly reduce transaction costs.

\section{CONCLUSION}

Today, the active development of professional interest in the digitalization of the economy in general and the introduction of blockchain technologies, including for the purpose of fixing rights to the results of intellectual activity and registration of legal relations on the use of the intellectual property can be noted. This phenomenon includes not only ways of a simple exchange of information but also the legal regime for formalizing the emergence, alteration, and termination of both real and obligation legal relations. Blockchain technologies applicable to the regulation of legal relations directly affect both the form and the content of the relevant legal relations. At the same time, digital technological novelties 
[5] S.V. Odintsov, M.V. Trubina, V.A. Koncheva, Tokens: actual problems of determining the legal status and classification. Scientific and Technical Revolution: Yesterday, Today and Tomorrow, Lecture Notes in Networks and Systems, Springer 2020, P. 787-790.

[6] Maher Alharby, Aad van Moorsel Blockchain-based smart contracts: a systematic mapping study. Computer Science and Information Technology, 2017. P. 125140 .

[7] Max Raskin, The Law and Legality of Smart Contracts. Georgetown Law Technology Review Vol 1:2, (2017), P. 305-340.

[8] UNCITRAL model law on electronic commerce, 1966

URL:https://uncitral.un.org/sites/uncitral.un.org/files/m edia-documents/uncitral/en/19-04970_ebook.pdf

[9] UNCITRAL model law on electronic signatures, 2001

URL:https://uncitral.un.org/sites/uncitral.un.org/files/m edia-documents/uncitral/en/ml-elecsig-e.pdf

\section{REFERENCES}

[1] M. Castells, The rise of the network society. With a New Preface. Vol. I. The Information Age: Economy, Society, and Culture. Publisher: Wiley-Blackwell, 2010, P. 78.

[2] M.N. Dudin, E.E. Frolova, O.V. Protopopova, A.A. Mamedov, S.V. Odintsov, Study of innovative technologies in the energy industry: nontraditional and renewable energy sources, Entrepreneurship and Sustainability Issues 6(4) 2019: P.1704-1713.

[3] V.A. Koncheva, S.V. Odintsov, L. Khmelnitski, Blockchain in HR, International scientific and practical digital economy conferences (ISCDE 2019), Atlantis Press, P. 787-790

[4] Bikramaditya Singhal, Gautam Dhameja, Priyansu Sekhar Panda Beginning Blockchain. A Beginner's Guide to Building Blockchain Solutions. Apress, 2018. P. 396. 